\title{
Research on New Battery System with Energy-Saving and Environment-Friendly Materials
}

\author{
Li Junming ${ }^{1}$, Zhang Fang ${ }^{1}$, Yu Xiaochen ${ }^{1}$, Su Hainan ${ }^{1}$, Yu Xin ${ }^{1}$, Pang Jing ${ }^{2 *}$, Xie Hongxu ${ }^{2}$ \\ ${ }^{1}$ Sate Grid Dandong Electric Power Supply Company, Dandong, Liaoning, 118000, China \\ ${ }^{2}$ Yantai Haibo Electrical Equipment Co., Ltd, Yantai, Shandong, 264001, China \\ (Note: The authors are ranked as follows: Li Junming is the first author, Zhang Fang is the second author, and Yu Xiaochen, Su Hainan, \\ Yu Xin, Pang Jing, and Xie Hongxu are the co-third authors)
}

\begin{abstract}
Applying the lithium iron phosphate battery online monitoring system to the DC power supply system of the substation is an innovative measure for energy saving and environmental protection of power enterprises. Nowadays, the world is advocating the use of energy-saving and environmentally-friendly green resources, which undoubtedly opens up a wider space for the research of lithium iron phosphate batteries. The article focuses on the performance analysis of the lithium iron phosphate battery system, the research significance, the composition of the system and the key technologies used.
\end{abstract}

\section{Introduction}

With the promotion and application of new technologies such as low-carbon economic technology and clean energy technology, new storage batteries made of energy-saving and environmentally-friendly materials are used in the operation and maintenance of DC power supply systems in substations. As a new type of secondary power supply, with its ultra-long life, safe use, environmental protection, high working voltage, high energy density, low self-discharge rate, no memory effect, and small size, it has great potential for the development of the secondary battery industry. New battery for energy saving and environmental protection materials is the future development direction of energy storage batteries.

\section{Comparison of battery performance}

\begin{tabular}{ccc}
\hline Project & lead-acid batteries & Lithium iron phosphate batteries \\
\hline Energy density $(\mathrm{Wh} / \mathrm{L})$ & 70 & 210 \\
Specific energy $\quad(\mathrm{mAh} / \mathrm{g})$ & $30-45$ & $110-165$ \\
Discharge rate & $0.1 \mathrm{C}-0.2 \mathrm{C}$ & from $2-5 \mathrm{C}$ to $10 \mathrm{C}$ \\
Cycle index & $300-400$ & $2000 \mathrm{above}$ \\
Working temperature & $-20-50^{\circ} \mathrm{C}$ & $-40-60^{\circ} \mathrm{C}$ \\
Balance & unbalanced function & balanced function \\
\hline
\end{tabular}

Compared with lead-acid batteries, lithium iron phosphate batteries have 3 times higher energy density, 5 times longer cycle times, and much higher specific energy. The discharge capacity of lithium iron phosphate battery is greater than $90 \%$ at $-40^{\circ} \mathrm{C}$, and it can still be discharged at full capacity at $55^{\circ} \mathrm{C}$ without affecting its service life. The lithium iron phosphate battery pack has its own BMS management system to manage and control the battery cells at any time to ensure that the battery pack is always kept in the best working condition.

Table 2. Comparison of technical properties of lithium Iron phosphate battery and lead acid battery

\begin{tabular}{|c|c|c|}
\hline Project & Lead-acid battery & Lithium iron phosphate battery \\
\hline Memory effect & Yes & No \\
\hline Discharge performance & $\begin{array}{c}\text { General } 70 \%-80 \% \\
\text { more than } 80 \% \text { damage to batteries }\end{array}$ & Full capacity discharge \\
\hline $\begin{array}{l}\text { Temperature compensation } \\
\text { Intelligentialize }\end{array}$ & $\begin{array}{l}\text { Temperature compensation is required } \\
\text { Not }\end{array}$ & $\begin{array}{l}\text { No required } \\
\text { Fully }\end{array}$ \\
\hline
\end{tabular}

*Corresponding author: tg667788@xzcstudio.com 


\begin{tabular}{|c|c|c|}
\hline Safety & Bad & Good \\
\hline Maintenance & Regular check charge/discharge & Maintenance-free \\
\hline Environmental protection & Toxic, polluting & Non-toxic, non-polluting \\
\hline Cost & High & Low \\
\hline \multicolumn{3}{|c|}{$\begin{array}{l}\text { Lithium iron phosphate batteries have no memory effect and can be discharged at any time and can be } \\
\text { fully discharged in any situation. Full-capacity discharge does not affect the life span, and it is not } \\
\text { affected by temperature without temperature compensation. Full-capacity discharge is } \\
\text { maintenance-free, safe and environmentally friendly with its own battery management system BMS, } \\
\text { which is completely intelligent and low in cost. }\end{array}$} \\
\hline
\end{tabular}

\section{Significance of researching new battery system with energy-saving and environmentally friendly materials}

In the substation, the battery pack of the DC system is connected in parallel with the charger to supply power to important DC loads such as relay protection, automatic devices, automatic equipment, and circuit breaker tripping and closing mechanisms. When AC power is lost, the motor cannot output DC power. The battery pack is the only DC power source to supply power to the DC load. Battery failure in an emergency situation may lead to major operating accidents in the substation. Therefore, whether the battery pack is normal or not directly affects the safe and reliable operation of the power system.

Traditional battery maintenance methods require inspectors to carry instruments to the site to conduct battery performance tests, record data and perform data analysis. This requires maintenance personnel to do a lot of testing work. Due to the large number of batteries and heavy workload in the DC power supply system, misoperation may sometimes cause a short circuit of the line during the on-site inspection process, which may harm the normal operation of the system. In view of the limited operation and maintenance personnel of the DC system, it is difficult to understand the operating conditions of each set of batteries in real time. After using the lithium iron phosphate battery system for online monitoring, it can reduce the potential safety hazards to the system due to the misoperation of the maintenance personnel.

\section{New battery system with energy-saving and environmentally friendly materials}

The new battery system of energy-saving and environmentally friendly materials is composed of batteries, battery management system (BMS) and protection circuits. The battery management system (BMS) is composed of the main control unit (CMU), the data acquisition unit (BMU), and the interface communication unit (ICU).

\subsection{System introduction}

The new battery system of energy-saving and environmentally-friendly materials adopts RS485 communication, which is more suitable for power systems and industrial and mining enterprises' substation DC systems, UPS, EPS and other backup power supply fields. It can realize the real-time control of the programmable DC power supply. Because the microprocessor has powerful sequential circuit processing capabilities and digital signal processing capabilities, it effectively improves the transient response and real-time performance of the programmable DC power supply. The temperature drift compensation circuit technology is adopted to ensure that the accuracy of the circuit meets the design requirements in a wide temperature range. Using digital PID control, the digital error control signal is directly converted into an output control signal through the DAC circuit, and the DAC circuit provides the output control signal to the isolated drive circuit to realize the real-time control of the programmable DC power supply. It can use TCP/IP protocol stack lwip technology to realize fast communication between PC and lithium battery BMS management system. The PC software communicates with multiple sets of BMS through the intranet, and transmits the battery voltage, current, remaining capacity SOC, health status $\mathrm{SOH}$, system status and other data to the PC software to display and save. It can use data transparent transmission cloud service technology, support SDK interface access technology of Andriod and IOS. Mobile phone APP can remotely view lithium battery pack data and charts. The lithium battery adopts the smallest module cold connection method, which not only avoids the problem of group replacement due to the problem of a single battery cell and reduces the replacement cost, but also shortens the replacement time as shown in figure 1, figure 2 . 


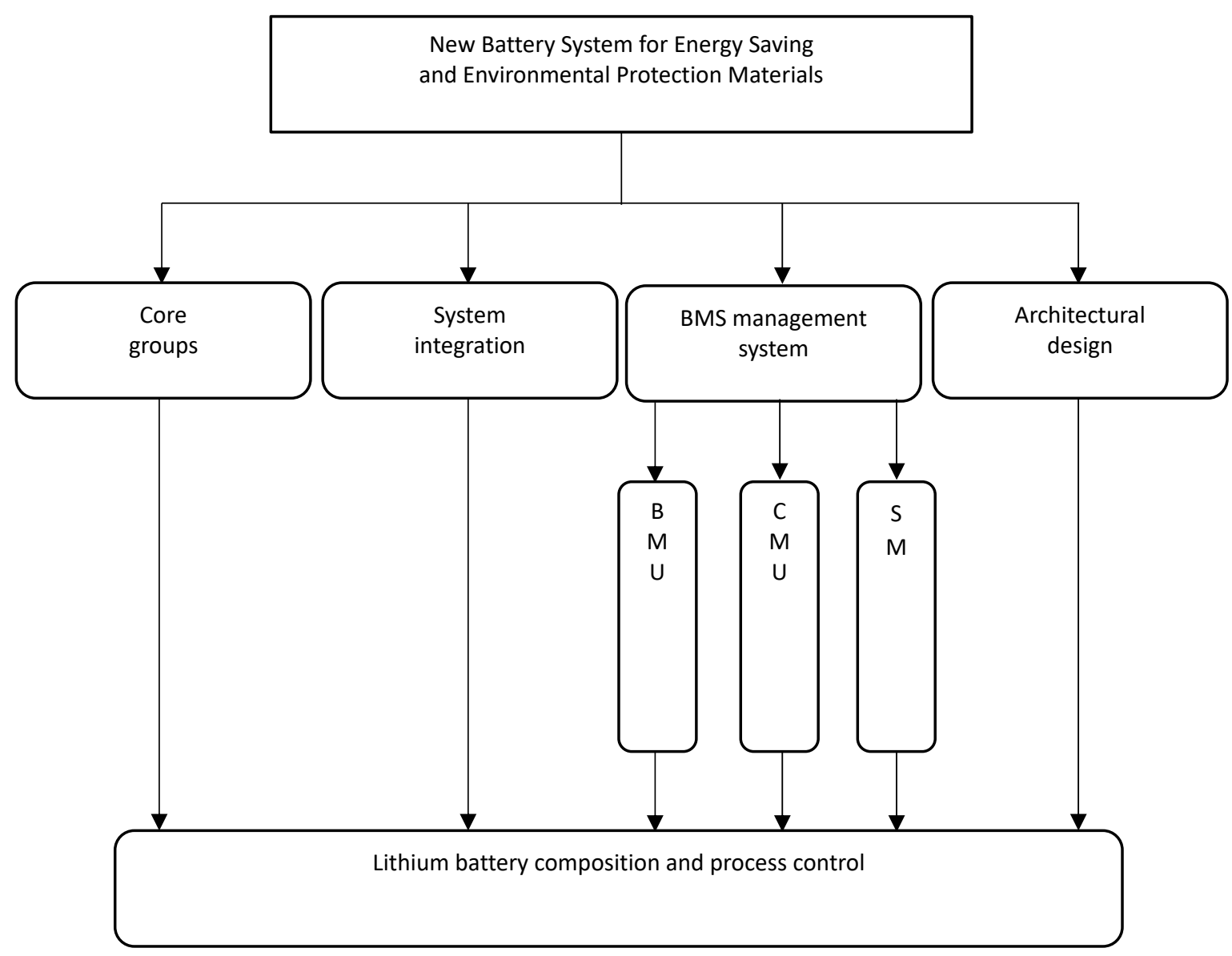

Figure 1. Flowchart of new battery system for energy saving and environmental protection materials

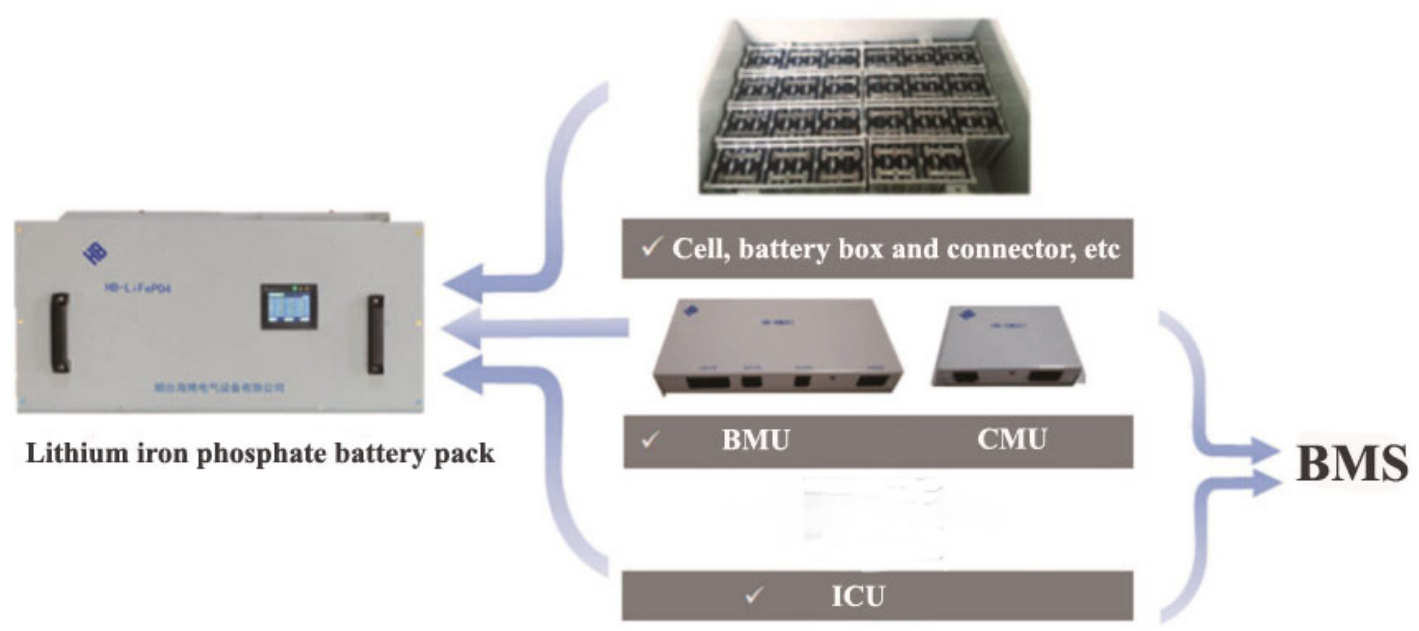

Figure 2. Composition of new storage battery system for energy saving and environmental protection materials

\subsection{Battery management system (BMS)}

The intelligent battery management system is the guarantee for the safe operation of the battery pack. It not only requires high-precision display, but also has various protection and alarm functions. Various communication interfaces have common protocols for power substation automation systems, and can also realize multiple industrial sites. It can realize centralized management of a set of lithium battery backup power system to achieve efficient management, graphical historical data, cloud services, remote maintenance, real-time monitoring of battery physical parameters, battery status estimation, online diagnosis and early warning, charge and discharge 
control, balance management and thermal management and other functions.

The battery management system consists of the main control unit $\mathrm{CMU}$, the data acquisition unit BMU, and the interface communication unit ICU, as shown in figure 3. Battery management system (BATTERY MANAGEMENT SYSTEM), the main components include main control unit $\mathrm{CMU}$, data acquisition unit BMU and interface communication unit ICU.

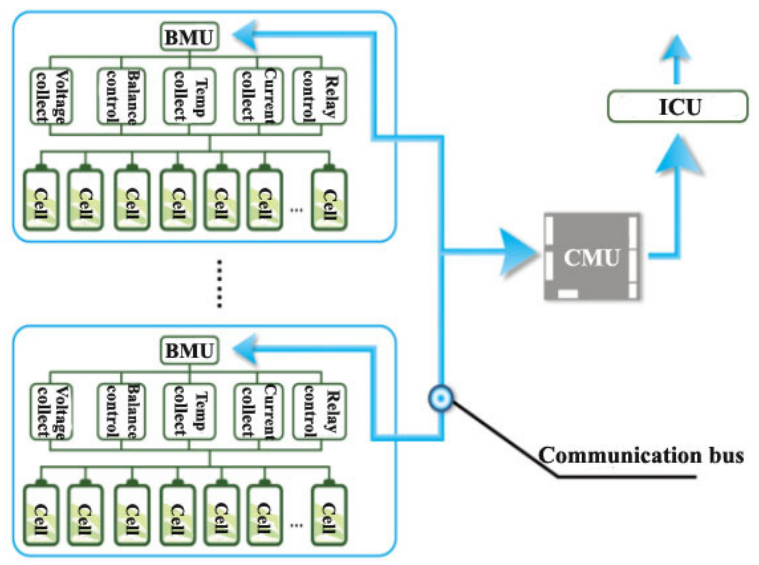

Figure 3. Structure chart of battery management system
The data acquisition unit of the management system is responsible for collecting the voltage, charge and discharge current, temperature and other data of each cell in the box, and perform balanced management and charge and discharge protection for the cells. The main control unit collects the data of the data acquisition unit, conducts centralized analysis and processing of the battery pack data, and performs alarm and control according to the operating conditions of the battery pack. At the same time, the main control unit also completes the measurement of the working current of the battery pack and the charge and discharge control.

\subsection{Main control unit (CUM)}

The main control unit CMU completes accurate dynamic estimation of SOC, monitors the current during the charging and discharging process of the battery pack, and monitors the current in real time, and displays various information of the battery pack in real time. When a fault occurs, the buzzer will give an alarm and display the specific fault type, realize the corresponding protection function through the control unit as shown in figure 4.

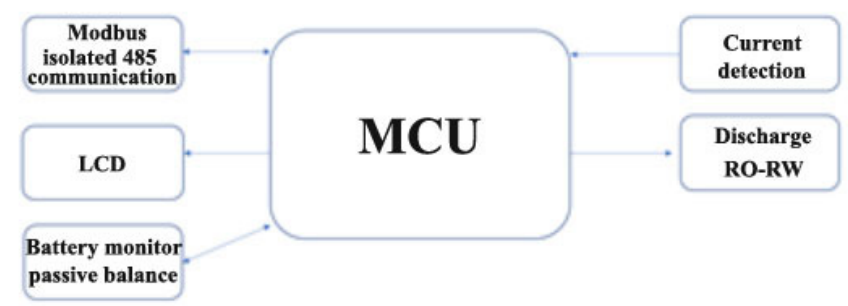

Figure 4. Main control unit function diagram

\subsection{Data acquisition unit (BMU)}

Each data acquisition unit can complete data acquisition, voltage equalization, and charge-discharge protection functions of 8 to 24 cells to maintain the consistency of the charging voltage of the parallel cells, thereby protecting all cells from damage during the charging process. At the same time, the data acquisition unit transmits the collected cell voltage, temperature and other information to the main control unit through the bus.

\subsection{Interface communications unit (ICU)}

The interface communication unit (ICU) is the human-computer interaction interface of the battery management system. It communicates through the RS485/CAN bus and can display various operating parameters and fault conditions of the system. All models are designed in accordance with industry standards and are suitable for use in various environments.

\section{Conclusion}

The new battery system with energy-saving and environmentally friendly materials completely solves the hidden safety hazards of various batteries in the past when they encountered strong impacts, drops, needle sticks, open flames, overcharge, and high temperatures. In the calculation of the entire life cycle of the entire substation battery, compared to the traditional lead-acid battery, the use of new energy-saving and environmentally-friendly materials can save more than $40 \%$ of the investment. The new type of energy-saving and environmentally-friendly material battery does not pollute the environment with a high recycling rate. It has a wide range of application prospects and promotion value in the DC system of substations, and the social benefits are very significant.

\section{Acknowledgment}

Name of Project Research: Application Research of New Battery Based on Energy Saving and Environmental 
Protection Material in Operation and Maintenance of DC Power Supply System in Substation

\section{References}

1. Zhao Xin, Zheng Lifeng, Deng Longzheng. Research and application status of lithium-ion power batteries and their key materials, J. New Materials Industry, 2006, 9: 44-47.

2. Zhang Xinhua, Tian Jun, Chen Hua. Heavy metal pollution in lead storage battery industry and countermeasures, J. Pollution Technology, 2013, 26(3): 33-35.

3. Li Jin, Zhang Yu, Li Jinglin. Feasibility analysis of the application of lithium iron phosphate batteries in substation systems, J. East China Electric Power, 2009, 37(10): 1693-1697.

4. Yang Jiayuan, Lai Qiu. Research on the application of lithium iron phosphate battery DC power supply system, J. Yunnan Electric Power Technology, 2011, 39(5): 45-47.

5. Wang Hong, Zhang Guanghui, Xing Jingyuan. Application research and practice of lithium iron phosphate batteries in substations, J. Power Technology, 2011, 35(8): 902-905. 